Ilchmann, Achim; Owens, David H.; Prätzel-Wolters, D. :

Sufficient conditions for stability of linear time-varying systems

Zuerst erschienen in:

Systems \& Control Letters 9 (1987), S. 157-163

DOI: 10.1016/0167-6911(87)90022-3 


\title{
Sufficient conditions for stability of linear time-varying systems
}

\author{
A. ILCHMANN \\ Institut für Dynamische Systeme, Universität Bremen, 2800 Bremen 33, West Germany \\ D.H. OWENS \\ Department of Mathematics, University of Strathclyde, Glasgow GI IXH, Scotland \\ D. PRÄTZEL-WOLTERS \\ Institut für Dynamische Systeme, Universität Bremen, 2800 Bremen 33, West Germany
}

Received 16 October 1986

Revised 18 February 1987

Abstract: In this paper we consider sufficient conditions for the exponential stability of linear time-varying systems of the form $\dot{x}(t)=A(t) x(t), t \geq 0$. Stability guaranteeing upper bounds for different measures of parameter variations are derived.

Keywords: Time-varying linear systems, Exponential stability.

\section{Introduction}

Stability analysis for time-varying linear systems is of increasing interest in control theory. One reason is the growing importance of adaptive controllers for which the underlying closed-loop adaptive system often is time-varying and linear.

In this paper we analyse exponential stability for systems of the form $\dot{x}(t)=A(t) x(t), t \geq 0$, where $A(\cdot): \mathbb{R}_{+} \rightarrow \mathbb{R}^{n \times n}$ is piecewise continuous and uniformly bounded. Furthermore for every $t \geq 0$ the eigenvalues of $A(t)$ are contained in a left half plane $\mathbb{C}^{-\varepsilon}=\{s \in \mathbb{C} \mid \operatorname{Re} s \leqslant-\varepsilon\}$ for some $\varepsilon>0$. However this last condition is not strong enough to guarantee exponential stability. Additional restrictions on the parameter variations in $A(\cdot)$ have to be imposed.

In Section 2 we summarize different types of those sufficient parameter variation conditions including the well known criteria of Coppel [2] and Rosenbrock [5] and two new conditions due to Kreisselmeier [4] and Krause and Kumar [3]. We give a new short proof of the Krause and Kumar result which was recently published in this journal. However all mentioned conditions are qualitative results in the sense that if some measure of the parameter variation is 'sufficiently small' the exponential stability is ensured.

In Section 3 we derive explicit formulas for the parameter variations upper bound to guarantee exponential stability. These formulas involve some a priori knowledge of $\|A(t)\|$ and $\sigma(A(t))$.

\section{Sufficient conditions for exponential stability}

To derive stability results for linear time-varying systems of the form

$$
\dot{x}(t)=A(t) x(t), \quad t \geq 0,
$$

it is usually a priori required that $A(\cdot)$ belongs to the set $\mathscr{S}$ of all piecewise continuous matrix functions

$$
A(\cdot): \mathbb{R}_{+} \rightarrow \mathbb{R}^{n \times n}
$$


which satisfy:

there exists $M>0$ such that $\|A(t)\| \leq M$ for all $t \geq 0$,

there exists $\alpha>0$ such that $\sigma(A(t)) \subset \mathbb{C}^{-\alpha}:=\{s \in \mathbb{C} \mid \operatorname{Re} s<-\alpha\}$ for all $t \geq 0$.

The following definition of exponential stability is standard:

2.1. Definition. A system (2.1) is called exponentially stable if there exist $L, \lambda>0$ such that

$$
\left\|\phi\left(t, t_{0}\right)\right\| \leq L \mathrm{e}^{-\lambda\left(t-t_{0}\right)} \text { for all } t \geq t_{0} \geq 0,
$$

where $\phi(\cdot, \cdot)$ denotes the transition matrix of $(2.1)$.

In general $A(\cdot) \in \mathscr{S}$ is neither necessary nor sufficient for exponential stability.

2.2. Examples. (i) Coppel [2,p.3]. Let

$$
A(t)=\left[\begin{array}{cc}
\cos t & -\sin t \\
\sin t & \cos t
\end{array}\right]\left[\begin{array}{rr}
-1 & -5 \\
0 & -1
\end{array}\right]\left[\begin{array}{cc}
\cos t & \sin t \\
-\sin t & \cos t
\end{array}\right] .
$$

Then $\sigma(A(t))=\{-1\}$ for all $t \geq 0$ and a calculation of a fundamental matrix shows that $A(\cdot)$ is unstable.

(ii) $\mathrm{Wu}[6]$. Let

$$
A(t)=\left[\begin{array}{cc}
-\frac{11}{2}+\frac{15}{2} \sin 12 t & \frac{15}{2} \cos 12 t \\
\frac{15}{2} \cos 12 t & -\frac{11}{2}-\frac{15}{2} \sin 12 t
\end{array}\right] .
$$
Then $\sigma(A(t))=\{2,-13\}$ for all $t \geq 0$; however the associated system $\dot{x}(t)=A(t) x(t)$ is exponentially
stable.

In order obtain sufficient conditions for exponential stability additional restrictions for the variation of the elements of $A(\cdot) \in \mathscr{S}$ have to be imposed.

It has been shown that if $\delta>0$ is sufficiently small, then any of the following conditions guarantees exponential stability of $(2.1)$ :

$$
\begin{aligned}
& \|\dot{A}(t)\| \leq \delta \quad \text { for all } t \geq 0 \quad[5] . \\
& \left\|A\left(t_{2}\right)-A\left(t_{1}\right)\right\| \leq \delta\left\|t_{2}-t_{1}\right\| \quad \text { for all } t_{1}, t_{2} \geq 0 \quad[2, \mathrm{p} .5] \\
& \sup _{0 \leq \tau \leq h}\|A(t+\tau)-A(t)\| \leq \delta \quad \text { for some } h>0
\end{aligned}
$$

$\dot{A}(\cdot)$ is continuous, $\|\dot{A}(\cdot)\|$ is uniformly bounded

and there exists $T>0$ such that $\int_{t_{0}}^{t_{0}+T}\|\dot{A}(t)\| \mathrm{d} t \leq \delta T$ for all $t_{0} \geq 0$.

Condition (2.7) is a consequence of Theorem 3.2 (iii) in Section 3 of this paper and is less restrictive than the similar condition in Lemma 3 of [4]:

$$
\lim _{t \rightarrow \infty} \sup _{0 \leq \tau \leq h}\|A(t+\tau)-A(t)\|=0 \quad \text { for all } h>0 .
$$

Furthermore (2.8) is less restrictive than the criterion in [3] which requires the integral inequality of (2.8) for all $T \geq T_{0}$ and some $T_{0}>0$. In fact condition (2.8) can be proved much shorter following the ideas of Rosenbrock's proof for condition (2.5).

'Simpler' proof of the Krause and Kumar condition. Let $A(\cdot) \in \mathscr{P}, \dot{A}(\cdot)$ continuous and uniformly bounded. Suppose the integral condition in (2.8) is satisfied for some $T>0, \delta>0$. Then the set

$$
\tau_{A}(\varepsilon):=\left\{t \in R_{+} \mid\|\dot{A}(\cdot)\|>\varepsilon\right\}
$$


is a union of open intervals since $\dot{A}(\cdot)$ is continuous. If $\mathscr{L}(I)$ denotes the Lebesgue measure of measurable sets $I \subset \mathbb{R}$, then by (2.8),

$$
\mathscr{L}\left(\left(t_{0}, t_{0}+T\right) \cap \tau_{A}(\varepsilon)\right) \cdot \varepsilon \leq \delta T \text { for all } t_{0} \geq 0 .
$$

Thus $\mathscr{L}\left(\left(t_{0}, t_{0}+T\right) \cap \tau_{A}(\varepsilon)\right) \rightarrow 0$ as $\delta \rightarrow 0$. Let now

$$
V(x, t):=x^{\mathrm{T}} R(t) x
$$

where

$$
R(t):=\int_{0}^{\infty} \mathrm{e}^{A^{\mathrm{T}}(t) s} \mathrm{e}^{A(t) s} \mathrm{~d} s .
$$

Similar to Rosenbrock's [5] proof we show that if $\delta$ is sufficiently small, then $V(x, t)$ is a Liapunov function for (2.1). Since $A(\cdot)$ is bounded there exist (cf. Brockett [1,p.203]) $c_{1}, c_{2}>0$ such that $c_{1} I_{n} \leq R(t) \leq c_{2} I_{n}$. Furthermore because $R(t) A(t)+A^{\mathrm{T}}(t) R(t)=-I_{n}$ we obtain

$$
\dot{R}(t)=\int_{0}^{\infty} \mathrm{e}^{A^{\mathrm{T}}(t) s}\left[R(t) \dot{A}(t)+\dot{A}^{\mathrm{T}}(t) R(t)\right] \mathrm{e}^{A(t) s} \mathrm{~d} s
$$

and for $\varepsilon$ sufficiently small,

$$
\begin{aligned}
\dot{V}(x(t), t) & =\left\langle x(t),\left[\dot{R}(t)-I_{n}\right] x(t)\right\rangle \\
& \leq-\frac{1}{2}\|x(t)\|^{2} \leq-\frac{1}{2 c_{2}} V(x(t), t) \quad \text { for } t \notin \tau_{A}(\varepsilon) .
\end{aligned}
$$

Since $\|\dot{A}(t)\| \leq K^{*}$ for some $K^{*}>0$,

$$
\dot{V}(x(t), t) \leq K\|x(t)\|^{2} \leq \frac{K}{c_{1}} V(x(t), t) \text { for } t \in \tau_{A}(\varepsilon)
$$

for some $K \geqslant K^{*}$. Since $\mathscr{L}\left(\tau_{A}(\varepsilon)\right) \rightarrow 0$ as $\delta \rightarrow 0$, there exists for $\delta$ sufficiently small $\omega>0$ such that

$$
\int_{t_{0}}^{t} \frac{\dot{V}(x(s), s)}{V(x(s), s)} \mathrm{d} s \leq-\omega\left(t-t_{0}\right) \quad \text { for all } t \geq t_{0}+T
$$

and this proves exponential decaying of the solutions of (2.1).

As an existence condition (2.8) is not really an improvement of (2.5). The above proof shows that if $\|\dot{A}(\cdot)\|$ is assumed to be uniformly bounded there always exists sufficiently small $\delta>0$ such that the average parameter variation condition (2.8) implies that $A(\cdot)$ satisfies Rosenbrock's criterion. The converse direction is obvious. Hence, viewed as existence conditions (2.8) and (2.5) are equivalent. In order to show that (2.8) is less conservative than (2.5), upper bounds for the integral in (2.8) should be derived which guarantee exponential stability of (2.1). In the next section we determine such quantitative bounds for the $\delta$ 's involved in (2.5)-(2.7).

\section{Upper bounds for parameter variations}

In order to prove the main result of this paper we need a lemma and some preparatory formulas:

3.1. Lemma [2]. Suppose $A(\cdot) \in \mathscr{P}$, in particular $\|A(t)\| \leq M$. Then for every $\varepsilon \in(0,2 M)$,

$$
\left\|\mathrm{e}^{A(t) \sigma}\right\| \leq(2 M / \varepsilon)^{n-1} \mathrm{e}^{(-\alpha+\varepsilon) \sigma} \quad \text { for all } \sigma, t \geq 0 \text {. }
$$


For fixed $t_{0} \in \mathbb{R}_{+},(2.1)$ can be rewritten in the form

$$
\dot{x}(t)=A\left(t_{0}\right) x(t)+\left[A(t)-A\left(t_{0}\right)\right] x(t), \quad t \geq 0,
$$

and for $x\left(t_{0}\right)=x_{0} \in \mathbb{R}^{n}$ its solution is given by

$$
x(t)=\mathrm{e}^{A\left(t_{0}\right)\left(t-t_{0}\right)} x_{0}+\int_{t_{0}}^{t} \mathrm{e}^{A\left(t_{0}\right)(t-s)}\left[A(s)-A\left(t_{0}\right)\right] x(s) \mathrm{d} s .
$$

Thus for $A(\cdot) \in \mathscr{S}$ Coppel's Lemma yields

$$
\begin{aligned}
\|x(t)\| \leq & \kappa_{\varepsilon} \mathrm{e}^{(-\alpha+\varepsilon)\left(t-t_{0}\right)}\left\|x_{0}\right\| \\
& +\kappa_{\varepsilon} \int_{t_{0}}^{t} \mathrm{e}^{(-\alpha+\varepsilon)(t-s)}\left\|A(s)-A\left(t_{0}\right)\right\|\|x(s)\| \mathrm{d} s \quad \text { for } t \geq t_{0},
\end{aligned}
$$

where

$$
\kappa_{\varepsilon}:=(2 M / \varepsilon)^{n-1} .
$$

Applying Gronwalls's Lemma to (3.3) gives

$$
\|x(t)\| \leq \kappa_{\varepsilon} \exp \left[(-\alpha+\varepsilon)\left(t-t_{0}\right)+\kappa_{\varepsilon} \int_{t_{0}}^{t}\left\|A(s)-A\left(t_{0}\right)\right\| \mathrm{d} s\right]\left\|x_{0}\right\| \text { for all } t \geq t_{0} .
$$

3.2. Theorem. Suppose $A(\cdot) \in \mathscr{S}$. Then

$$
\dot{x}(t)=A(t) x(t), \quad t \geq 0,
$$

is exponentially stable if one of the following conditions holds for all $t \geq 0$ :

(i) $\alpha>4 M$.

(ii) $A(\cdot)$ is piecewise differentiable and

$$
\|\dot{A}(t)\| \leq \delta<\frac{2}{2 n-1} \frac{\alpha^{4 n-2}}{2 M^{4 n-4}} .
$$

(iii) For some $k \geq 0, \eta \in(0,1), \alpha>2 M \eta+((n-1) / k) \log \eta$ and

$$
\sup _{0 \leq \tau \leq k}\|A(t+\tau)-A(t)\| \leq \delta<\eta^{n-1}\left(\alpha-2 M \eta+\frac{n-1}{k} \log \eta\right) .
$$

(iv) $\alpha>n-1$ and for some $\eta \in(0,1)$,

$$
\sup _{h>0}\left\|\frac{A(t+h)-A(t)}{h}\right\| \leq \delta<2 \eta^{n-1}(\alpha-2 M \eta+(n-1) \log \eta) .
$$

Proof. (i) Since $\left\|A(s)-A\left(t_{0}\right)\right\| \leq 2 M \forall s, t_{0} \geq 0$, inequality (3.5) implies for some $h>0$ and $\varepsilon \in(0,2 M)$, $\|x(t)\| \leq \kappa_{\varepsilon} \mathrm{e}^{\left[-\alpha+\varepsilon+\kappa_{\varepsilon} 2 M\right]\left(t-t_{0}\right)}\left\|x\left(t_{0}\right)\right\| \leq \kappa_{\varepsilon} \mathrm{e}^{\left[\varepsilon+\kappa_{\varepsilon} 2 M-4 M-h\right]}\left\|x\left(t_{0}\right)\right\|$.

The function

$$
f:(0,2 M] \rightarrow R, \quad \varepsilon \mapsto \varepsilon+\kappa_{\varepsilon} 2 M-4 M-h,
$$

is continuous and $f(2 M)=-h$. Thus there exists $\varepsilon \in(0,2 M]$ such that $f(\varepsilon)<0$.

(ii) We prove that $V(x, t)$ as defined in (2.9) is a Liapunov function of (3.6). Its time derivative along solutions of (3.6) is

$$
\frac{\mathrm{d}}{\mathrm{d} t} V(x(t), t)=x^{\mathrm{T}}(t)\left[-I_{n}+\dot{R}(t)\right] x(t) .
$$


We have to show that

$$
\dot{R}(t)<I_{n} \text { for all } t \geq 0 \text {. }
$$

Applying Coppel's Lemma to (2.11) and (2.10) we obtain

$$
\begin{aligned}
\|\dot{R}(t)\| & \leq \int_{0}^{\infty}\left(\frac{2 M}{\varepsilon}\right)^{2(n-1)} \mathrm{e}^{2(-\alpha+\varepsilon) s} \mathrm{~d} s \cdot 2\|R(t)\|\|\dot{A}(t)\| \\
& \leq 2\left[\left(\frac{2 M}{\varepsilon}\right)^{2(n-1)} \int_{0}^{\infty} \mathrm{e}^{2(-\alpha+\varepsilon) s} \mathrm{~d} s\right]^{2} \delta=2\left(\frac{2 M}{\varepsilon}\right)^{4(n-1)}\left(\frac{1}{2(-\alpha+\varepsilon)}\right)^{2} \delta
\end{aligned}
$$

and thus (3.7) holds if for some $\varepsilon \in(0, \alpha)$,

$$
\delta<2\left(\frac{\varepsilon}{2 M}\right)^{4(n-1)}(\alpha-\varepsilon)^{2}=: g(\varepsilon) .
$$

It is easily verified that $g(\cdot)$ achieves its maximum at $\varepsilon_{0}=\alpha k /(k+1)$ on $(0, \alpha)$ and

$$
g\left(\varepsilon_{0}\right)=\frac{2 \alpha^{4 n-2}}{(2 M)^{4(n-1)}(n-1)} .
$$

(iii) By (3.5) we have for every $l \in \mathbb{N}, k>0$ and $t \in\left[t_{0}+l k, t_{0}+(l+1) k\right]$,

$$
\begin{aligned}
\|x(t)\| & =\kappa_{\varepsilon} \mathrm{e}^{\gamma\left(t-t_{0}-l k\right)}\left\|x\left(t_{0}+l k\right)\right\| \\
& \leq \kappa_{\varepsilon} \mathrm{e}^{\gamma\left(t-t_{0}-l k\right)} \kappa_{\varepsilon} \mathrm{e}^{\gamma(k)}\left\|x\left(t_{0}+(l-1) k\right)\right\|=\kappa_{\varepsilon}^{2} \mathrm{e}^{\gamma\left(t-t_{0}-(l-1) k\right)}\left\|x\left(t_{0}+(l-1) k\right)\right\| \\
& \leq \cdots \leq \kappa_{\varepsilon}^{l+1} \mathrm{e}^{\gamma\left(t-t_{0}\right)}\left\|x\left(t_{0}\right)\right\|
\end{aligned}
$$

where $\gamma:=-\alpha+\varepsilon+\kappa_{\varepsilon} \delta$. Thus

$$
\|x(t)\| \leq \kappa_{\epsilon} \mathrm{e}^{l \log \kappa_{\epsilon}+\gamma\left(t-t_{0}\right)}\left\|x\left(t_{0}\right)\right\| .
$$

Because $t-t_{0} \geq l k$ we obtain

$$
\|x(t)\| \leq \kappa_{\varepsilon} \exp \left\{\left(\frac{\log \kappa_{\varepsilon}}{k}+\gamma\right)\left(t-t_{0}\right)\right\}\left\|x\left(t_{0}\right)\right\| .
$$

\section{Consider}

$$
\frac{\log \kappa_{\varepsilon}}{k}+\gamma=\frac{\log (2 M / \varepsilon)^{n-1}}{k}+\varepsilon+\kappa_{\varepsilon} \delta-\alpha .
$$

It suffices to find $\varepsilon<2 M, k>0$ such that $\left(\log \kappa_{\varepsilon}\right) / k+\gamma<0$ or equivalently,

$$
(\varepsilon-\alpha)+\frac{\log \kappa_{\varepsilon}}{k}<-\kappa_{\varepsilon} \delta,
$$

respectively

$$
0<\delta<\frac{1}{\kappa_{\varepsilon}}\left(\alpha-\varepsilon-\frac{\log \kappa_{\varepsilon}}{k}\right) .
$$

However to every $\varepsilon \in(0, \alpha)$ exists $k^{*}>0$ such that $\alpha-\varepsilon-\left(\log \kappa_{\varepsilon}\right) / k^{*}>0$ and we obtain exponential stability of (3.6) for every $A(\cdot)$ for which

$$
\sup _{0 \leq \tau \leq k^{*}}\|A(t+\tau)-A(t)\| \leq \delta<\frac{1}{\kappa_{\varepsilon}}\left(\alpha-\varepsilon-\frac{\log \kappa_{\varepsilon}}{k^{*}}\right)>0
$$

Now (iii) follows with $\eta=\varepsilon / 2 M$. 
(iv) Assume

$$
\left\|\frac{A(t+h)-A(t)}{h}\right\| \leq \delta
$$

for every $h>0$. Then by (3.5) we have

$$
\begin{aligned}
\|x(t)\| & \leq \kappa_{\varepsilon} \exp \left\{(-\alpha+\varepsilon)\left(t-t_{0}\right)+\kappa_{3} \int_{0}^{t-t_{0}} h \delta \mathrm{d} h\right\}\left\|x\left(t_{0}\right)\right\| \\
& \leq \kappa_{\varepsilon} \exp \left\{\left(\varepsilon-\alpha+\kappa_{\varepsilon} \frac{t-t_{0}}{2} \delta\right)\left(t-t_{0}\right)\right\}\left\|x\left(t_{0}\right)\right\| .
\end{aligned}
$$

For $t \in\left[t_{0}, t_{0}+1\right]$ we obtain

$$
\|x(t)\| \leq \kappa_{\varepsilon} \mathrm{e}^{\gamma\left(t-t_{0}\right)}\left\|x\left(t_{0}\right)\right\|
$$

where $\gamma=\varepsilon-\alpha+\frac{1}{2} \kappa_{\varepsilon} \delta$. For $t \in\left[t_{0}+l, t_{0}+l+1\right], l \in \mathbb{N}$ arbitrary, we conclude as in the proof of (iii),

$$
\|x(t)\| \leq \kappa_{\varepsilon} \mathrm{e}^{\left(\log \kappa_{e}+\gamma\right)\left(t-t_{0}\right)}\left\|x\left(t_{0}\right)\right\|
$$

and $\log \kappa_{\varepsilon}+\gamma<0$ if

$$
\delta<2\left(\frac{\varepsilon}{2 M}\right)^{n-1}\left(\alpha-\varepsilon-\log \left(\frac{2 M}{\varepsilon}\right)^{n-1}\right) .
$$

Then (iv) follows with $\eta=\varepsilon / 2 M$.

Note that the proof of (iii) presents a short proof of Lemma 3 in Kreisselmeier [4].

If additional information on the exponential decay of $\mathrm{e}^{A(t) \tau}$ is known, the bounds derived in Theorem 3.2 can be simplified as follows:

3.3. Theorem. Suppose $A(\cdot) \in \mathscr{S}$ and let

$$
\left\|\mathrm{e}^{A(t) \sigma}\right\| \leq \kappa \mathrm{e}^{-\omega \sigma} \quad \text { for all } t, \sigma \geq 0,
$$

for some $\kappa, \omega>0$. Let $\beta:=\omega / \kappa$. Then the system

$$
\dot{x}(t)=A(t) x(t), \quad t \geq 0,
$$

is exponentially stable if one of the following conditions holds for all $t \geq 0$ :

(i) $\|A(t)\| \leq M<\frac{1}{2} \beta$.

(ii) $A(\cdot)$ is piecewise differentiable and

$$
\|\dot{A}(t)\| \leq \delta<2 \beta^{2} \text {. }
$$

(iii) There exists $h>0$ such that

$$
\sup _{0 \leq \tau \leq h}\|A(t+\tau)-A(t)\| \leq \delta<\beta-\frac{1}{h} \frac{\log \kappa}{\kappa} .
$$

(iv) $\sup _{h>0}\left\|\frac{A(t+h)-A(t)}{h}\right\| \leq \delta<2 \beta-2 \frac{\log \kappa}{\kappa}$.

The proof is similar to that of Theorem 3.2. 


\section{References}

[1] R.W. Brockett, Finite Dimensional Linear Systems (John Wiley, New York, 1970).

[2] W.A. Coppel, Dichotomies in Stability Theory, Lecture Notes in Mathematics No. 629 (Springer, Berlin-New York, 1978)

[3] J.M. Krause and K.S.P. Kumar, An atternative stability analysis framework for adaptive control, Systems Control Lett. 7 (1986) 19-24.

[4] G. Kreisselmeier, An approach to stable indirect adaptive control, Automatica 21 (9) (1985) 425-431.

[5] H.H. Rosenbrock, The stability of linear time-dependent control systems, Internat. J. Electr. Control 15 (1) (1963) 73-80.

[6] M.Y. Wu, A note on stability of linear time-varying systems, IEEE Trans. Automat. Control 19 (1974) 162. 